\title{
Semilinear Evolution Problems with Ventcel-Type Conditions on Fractal Boundaries
}

\author{
Maria Rosaria Lancia ${ }^{1}$ and Paola Vernole ${ }^{2}$ \\ ${ }^{1}$ Dipartimento di Scienze di Base e Applicate per l'Ingegneria, Università degli Studi di Roma "La Sapienza", \\ Via A. Scarpa 16, 00161 Roma, Italy \\ ${ }^{2}$ Dipartimento di Matematica, Università degli Studi di Roma “La Sapienza”, Piazzle Aldo Moro 2, 00185 Roma, Italy
}

Correspondence should be addressed to Maria Rosaria Lancia; maria.lancia@sbai.uniromal.it

Received 30 April 2013; Accepted 17 October 2013; Published 22 January 2014

Academic Editor: William E. Fitzgibbon

Copyright (C) 2014 M. R. Lancia and P. Vernole. This is an open access article distributed under the Creative Commons Attribution License, which permits unrestricted use, distribution, and reproduction in any medium, provided the original work is properly cited.

A semilinear parabolic transmission problem with Ventcel's boundary conditions on a fractal interface $S$ or the corresponding prefractal interface $S_{h}$ is studied. Regularity results for the solution in both cases are proved. The asymptotic behaviour of the solutions of the approximating problems to the solution of limit fractal problem is analyzed.

\section{Introduction}

In this paper we study the parabolic semilinear second-order transmission problem which we formally state as

$$
(\bar{P}) \begin{cases}u_{t}(t, P)-\Delta u(t, P)=J(u(t, P)) & \text { in }[0, T] \times Q^{i}, \\ -c_{0} \Delta_{L} u(t, P)=\left[\frac{\partial u(t, P)}{\partial n}\right] & \text { on }[0, T] \times L, \\ u(t, P)=0 & \text { on }[0, T] \times \partial Q \\ u^{1}(t, P)=u^{2}(t, P) & \text { on }[0, T] \times L, \\ u(t, P)=0 & \text { on }[0, T] \times \partial L \\ u(0, P)=\phi & \text { on } Q\end{cases}
$$

where $Q$ is the bounded open set $(-1,1)^{2} \times(0,1)$, and $L$ is a "cylindrical" layer dividing the set $Q$ into two subsets $Q^{1}$ and $Q^{2}$ (see Figure 2). When $L$ is the Koch-type surface $S=K \times I$, where $K$ is the snowflake and $I=[0,1]$ (see Section 2), $E_{L}$ is the energy functional $E_{S}$ introduced in (12); when $L$ is the prefractal surface $S_{h}, E_{L}$ is the energy functional $E_{S_{h}}$ introduced in (24). $J$ is a nonlinear function from a subset of $L^{2}(Q)$ into $L^{2}(Q) . u^{i}$ denotes the restriction of $u$ to $Q^{i}$, $[u]=u^{1}-u^{2}$ denotes the jump of $u$ across $L, \Delta_{L}$ denotes the Laplace operator defined on the layer $L$ (see (12) in Section 3), and $[\partial u / \partial n]=\partial u^{1} / \partial n_{1}+\partial u^{2} / \partial n_{2}$ denotes the jump of the normal derivatives across $L$, to be intended in a suitable sense.

More precisely, we assume that $J(u)$ is a nonlinear mapping from $L^{2 p}(Q)$ to $L^{2}(Q)$ for any fixed $p>1$, locally Lipschitz; that is, Lipschitz on bounded sets in $L^{2 p}(Q)$ with Lipschitz constant $l(r)$ when restricted to $B(0, r) \subset L^{2 p}(Q)$, satisfying suitable growth conditions (see conditions (i) and (ii) in Section 4). Examples of this type of nonlinearity include, for example, $J(u)=u|u|^{p-1}, p>1$ which occur in combustion theory (see [1]) and in the Navier-Stokes system (see [2]).

In the recent years there has been an increasing interest in the study of linear transmission problems across irregular layers of fractal type and the corresponding prefractal layers [3-7]. Problems of this type are also known in the literature as problems with Ventcel's boundary conditions [8] or secondorder transmission conditions. Fractal layers can provide new interesting settings in those model problems, in which the surface absorption of tension, electric conduction, or flow is the relevant effect. The literature on semilinear equations on smooth domains is extensive (see e.g., [9-13] and the recent review in [14]); the fractal case is more awkward (see e.g., [1519]).

In our case one has to take into account that the diffusion phenomenon takes place both across the smooth domain 
$Q$ and the cylindrical layer $L$; this fact has a counterpart in the structure of the energy functional $E[u]$ and hence on problem $(\bar{P})$. In [18] the authors proved local existence and uniqueness results of the "mild" solution of an abstract evolution transmission problem across a prefractal or fractal interface (see (36) and (37)).

In this paper we give a strong interpretation of the abstract problem studied in [18],;namely, we prove that the solution of the abstract problem solves problem $(\bar{P})$ in a suitable sense (see Theorems 22 and 20).

The results on the strong interpretation in the prefractal case are deduced by proving regularity results for the solutions of elliptic problems in polyhedral domains. It turns out that the restriction $u_{h}^{i}$ of the solution $u_{h}$ to $Q_{h}^{i}$ belongs to suitable weighted Sobolev spaces (see the proof of Theorem 22). This regularity result is important not only in itself but also in the numerical approximation procedure; to this regard, see [20]. Following this point of view, it is also important to study the asymptotic behaviour of the solutions of the prefractal problems.

The proof of the convergence of the solution of the prefractal problems to the one of the (limit) fractal problem relies on the convergence, in the Mosco's sense, of the energy forms which, in turn, implies the convergence of semigroups in the strong operator topology of $L^{2}(Q)$ (see Theorem 16). The plan of the paper is as follows. In Section 2 we describe the geometry of the problem; in Section 3 we introduce the Dirichlet energy forms and the associated semigroups and we recall the results on the convergence of the approximating energy forms (see [21] for details). In Section 4 we recall existence and uniqueness results for the local mild solution as well as global existence and regularity results. In Section 5 we prove that the solution of the abstract Cauchy problems $(P)$ and $\left(P_{h}\right)$ solves problem $(\bar{P})$ in the fractal and prefractal cases, respectively, (see Theorems 22 and 20). In Section 6 we prove the convergence of the solutions of the approximating problems to the solution of the limit fractal problem in a suitable functional space. In Appendices $\mathrm{A}$ and $\mathrm{B}$, for the reader convenience, we introduce the functional spaces and traces involved.

\section{Geometry of the Fractal Layers $S$ and $S_{h}$}

In the paper by $\left|P-P_{0}\right|$ we denote the Euclidean distance in $\mathbb{R}^{D}$ and the Euclidean balls by $B\left(P_{0}, r\right)=\left\{P \in \mathbb{R}^{D}:\left|P-P_{0}\right|<\right.$ $r\}, P_{0} \in \mathbb{R}^{D}, r>0$. By the Koch snowflake $F$, we will denote the union of three coplanar Koch curves (see [22]) $K_{1}, K_{2}$, and $K_{3}$ as shown in Figure 1. We assume that the junction points $A_{1}, A_{3}$, and $A_{5}$ are the vertices of a regular triangle with unit side length; that is, $\left|A_{1}-A_{3}\right|=\left|A_{1}-A_{5}\right|=\left|A_{3}-A_{5}\right|=1$. In this section we briefly recall the essential notions on the geometry; for details see [18].

The Hausdorff dimension of the Koch snowflake is given by $d_{f}=\log 4 / \log 3$. This fractal is no longer self-similar (and hence not nested).

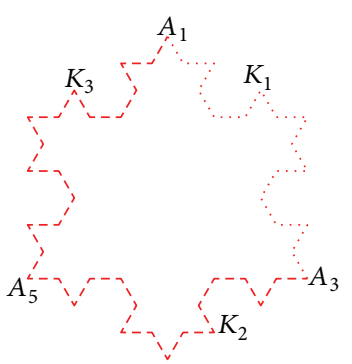

Figure 1: Decomposition of the snowflake.

One can define, in a natural way, a finite Borel measure $\mu_{F}$ supported on $F$ by

$$
\mu_{F}:=\mu_{1}+\mu_{2}+\mu_{3}
$$

where $\mu_{i}$ denotes the normalized $d_{f}$-dimensional Hausdorff measure, restricted to $K_{i}, i=1,2,3$.

The measure $\mu_{F}$ has the property that there exist two positive constants $c_{1}$ and $c_{2}$ :

$$
c_{1} r^{d} \leq \mu_{F}(B(P, r) \cap F) \leq c_{2} r^{d}, \quad \forall P \in F,
$$

where $d=d_{f}=\log 4 / \log 3$ and $B(P, r)$ denotes the Euclidean ball in $\mathbb{R}^{2}$. As $\mu_{F}$ is supported on $F$, it is not ambiguous to write in $(3) \mu_{F}(B(P, r))$ in place of $\mu_{F}(B(P, r) \cap F)$. In the terminology of Appendices $\mathrm{A}$ and $\mathrm{B}$, we say that $F$ is a $d$-set with $d=d_{f}$.

Remark 1. The Koch snowflake can be also regarded as a fractal manifold (see [23] Section 2.2).

Let $Q$ denote a bounded open set in $\mathbb{R}^{3}$; in our basic model, $Q$ denotes the parallelepiped $Q=(-1,1)^{2} \times(0,1)$ and $S$ denotes a "cylindrical" layer in $Q$ of the type $S=F \times I$, where $I=[0,1]$ and $F$ is the Koch snowflake. We assume that $S$ is located in a median position inside $Q$ and divides $Q$ in two subsets $Q^{1}$ and $Q^{2}$ (see Figure 2).

We give a point $P \in S$ the Cartesian coordinates $P=(x, y)$, where $x=\left(x_{1}, x_{2}\right)$ are the coordinates of the orthogonal projection of $P$ on the plane containing $F$ and $y$ is the coordinate of the orthogonal projection of $P$ on the $y$-line containing the interval $I: P=(x, y) \in S, x=\left(x_{1}, x_{2}\right) \in F$, $y \in I$.

One can define, in a natural way, a finite Borel measure $m$ supported on $S$ as the product measure

$$
d m=d \mu_{F} d y,
$$

where $d y$ denotes the one-dimensional Lebesgue measure on $I$. The measure $m$ has the property that there exist two positive constants $c_{1}$ and $c_{2}$ :

$$
c_{1} r^{d} \leq m(B(P, r) \cap S) \leq c_{2} r^{d}, \quad \forall P \in S,
$$

where $d=d_{f}+1=\log 12 / \log 3$ and $B(P, r)$ denotes the Euclidean ball in $\mathbb{R}^{3}$. As $m$ is supported on $S$, it is not ambiguous to write in $(5) m(B(P, r))$ in place of $m(B(P, r) \cap S)$. 


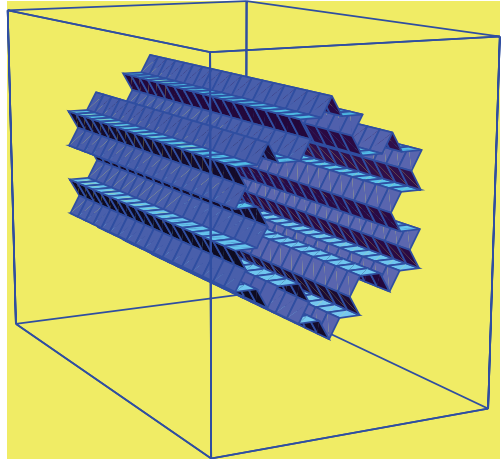

(a)

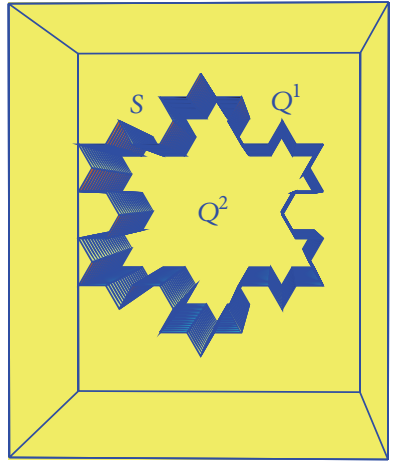

(b)

FIgURE 2: Two different viewpoints of the domain $Q$ and the layer $S$.

Thus $S$ turns out to be a $d$-set with $d=d_{f}+1$ (see Appendices $\mathrm{A}$ and $\mathrm{B})$.

By $S_{h}$, we denote the prefractal layer of the type $S_{h}=$ $F_{h} \times I, h=1,2, \ldots, F_{h}$ is the piecewise linear prefractal approximation of $F$ at the step $h . S_{h}$ is a surface of polyhedral type. $S_{h}$ divides $Q$ in two subsets $Q_{h}^{i}, i=1,2$.

We give a point $P \in S_{h}$ the Cartesian coordinates $P=(x, y)$, where $x=\left(x_{1}, x_{2}\right)$ are the coordinates of the orthogonal projection of $P$ on the plane containing $F_{h}$ and $y$ is the coordinate of the orthogonal projection $P$ on the $y$-line containing the interval $I$.

\section{Energy Forms and Semigroups Associated}

3.1. The Energy Form E. In this section we introduce the energy functional on $S$. We first define the energy functional on the cross section $F$ by integrating its Lagrangian on $F$. For the concept of Lagrangian on fractals, that is, the notion of a measure-valued local energy, we refer to [24, 25]. Here for the sake of simplicity we only mention that the Lagrangian on $K, \mathscr{L}_{K}$, is a measure-valued map on $\mathscr{D}(F) \times$ $\mathscr{D}(F)$ which is bilinear symmetric and positive $\left(\mathscr{L}_{K}[u]\right.$ is a positive measure.) The measure-valued Lagrangian takes on the fractal $K$ the role of the Euclidean Lagrangian $d \mathscr{L}(u, v)=$ $\nabla u \cdot \nabla v d x$. Note that in the case of the Koch curve, the Lagrangian is absolutely continuous with respect to the measure $\mu$; on the contrary, this is not true on most fractals (see [24]). In [23] the Lagrangian $\mathscr{L}_{F}$ on the snowflake $F$ has been defined by using its representation as a fractal manifold. Here we do not give details on the construction and definition of $\mathscr{L}_{F}$ and we refer to Section 4 in [23] for details; in particular in Definition 4.5 a Lagrangian measure $\mathscr{L}_{F}$ on $F$ and the corresponding energy form $\mathscr{E}_{F}$ as

$$
\mathscr{E}_{F}(u, v)=\int_{F} d \mathscr{L}_{F}(u, v)
$$

with domain $\mathscr{D}(F)$ have been introduced. The domain $\mathscr{D}(F)$, which is a Hilbert space with norm

$$
\left(\|u\|_{L^{2}\left(F, \mu_{F}\right)}^{2}+\mathscr{E}_{F}(u, u)\right)^{1 / 2}
$$

has been characterized in terms of the domains of the energy forms on $K_{i}$ (see [23] Theorem 4.6).

In the following, we will omit the subscript $F$, the Lagrangian measure will be simply denoted by $\mathscr{L}(u, v)$, and we will set $\mathscr{L}[u]=\mathscr{L}(u, u)$; an analogous notation will be adopted for the energies.

We define the energy forms $E_{S}$ on the fractal layer $S=$ $F \times I$ by setting

$$
\begin{aligned}
E_{S}[u]= & \sigma^{1} \int_{I} \int_{F} \mathscr{L}_{x}[u](d x) d y \\
& +\sigma^{2} \int_{F} \int_{I}\left|D_{y} u\right|^{2} d y \mu_{F}(d x),
\end{aligned}
$$

where $\sigma^{1}$ and $\sigma^{2}$ are positive constants. Here $\mathscr{L}_{x}(\cdot, \cdot)(d x)$ denotes the measure-valued Lagrangian (of the energy form $\mathscr{E}_{F}$ of $F$ with domain $\left.\mathscr{D}(F)\right)$ now acting on $u(x, y)$ and $v(x, y)$ as function of $x \in F$ for a.e. $y \in I ; \mu_{F}(d x)$ is the $d_{f^{-}}$ Hausdorff measure acting on each section $F$ of $S$ for a.e. $y \in I$ with $d_{f}=\log 4 / \log 3 ; D_{y}(\cdot)$ denotes the derivative in the $y$ direction.

The form $E_{S}$ is defined for $u \in \mathscr{D}(S)$, where $\mathscr{D}(S)$ is the closure in the intrinsic norm

$$
\|u\|_{\mathscr{D}(S)}=\left(E_{S}[u]+\|u\|_{L^{2}(S, m)}^{2}\right)^{1 / 2}
$$

of the set

$$
C_{0}(S) \cap L^{2}((0,1) ; \mathscr{D}(F)) \cap H_{0}^{1}\left((0,1) ; L^{2}(F)\right),
$$

where $L^{2}(F)=L^{2}\left(F, \mu_{F}(d x)\right)$.

In the following, we will also use the form $E_{S}(u, v)$ which is obtained from $E_{S}[u]$ by the polarization identity:

$$
\begin{array}{r}
E_{S}(u, v)=\frac{1}{2}\left\{E_{S}[u+v]-E_{S}[u]-E_{S}[v]\right\}, \\
u, v \in \mathscr{D}(S) .
\end{array}
$$

Proposition 2. In the previous notations and assumptions, the form $E_{S}$ with domain $\mathscr{D}(S)$ is a regular Dirichlet form in $L^{2}(S, m)$ and the space $\mathscr{D}(S)$ is a Hilbert space under the intrinsic norm (9). 
The proof can be carried on as in Proposition 3.1 of [26]. For the definition and properties of regular Dirichlet forms, we refer to [25]. We now define the Laplace operator on $S$. As $\left(E_{S}, \mathscr{D}(S)\right)$ is a closed, bilinear form on $L^{2}(S, m)$, there exists (see Chapter 6, Theorem 2.1 in [27]) a unique selfadjoint, nonpositive operator $\Delta_{S}$ on $L^{2}(S, m)$-with domain $\mathscr{D}\left(\Delta_{S}\right) \subseteq \mathscr{D}(S)$ dense in $L^{2}(S, m)$-such that

$$
\begin{gathered}
E_{S}(u, v)=-\int_{S}\left(\Delta_{S} u\right) v d m, \\
u \in \mathscr{D}\left(\Delta_{S}\right), \quad v \in \mathscr{D}(S) .
\end{gathered}
$$

Let $(\mathscr{D}(S))^{\prime}$ denote the dual of the space $\mathscr{D}(S)$. We now introduce the Laplace operator on the fractal $S$ as a variational operator from $\mathscr{D}(S) \rightarrow(\mathscr{D}(S))^{\prime}$ by

$$
E_{S}(z, w)=-\left\langle\Delta_{S} z, w\right\rangle_{(\mathscr{D}(S))^{\prime}, \mathscr{D}(S)}
$$

for $z \in \mathscr{D}(S)$ and for all $w \in \mathscr{D}(S)$, where $\langle\cdot, \cdot\rangle_{(\mathscr{D}(S))^{\prime}, \mathscr{D}(S)}$ is the duality pairing between $(\mathscr{D}(S))^{\prime}$ and $\mathscr{D}(S)$. We use the same symbol $\Delta_{S}$ to define the Laplace operator both as a selfadjoint operator in (12) and as a variational operator in (13). It will be clear from the context to which case we refer.

In the next, we will also use the spectral dimension $v$ of $S$. We find that if $r(\lambda)$ is the number of eigenvalues associated with $E_{S}$ smaller than $\lambda$, then $r(\lambda) \sim \lambda^{\nu / 2}$. It can be shown that in our case $v=2$ (see $[28,29])$. We stress the fact that in the fractal case $v<d<D$, while in the Euclidean setting $v=d$.

Consider now the space of functions $u: Q \rightarrow \mathbb{R}$ as

$$
V(Q, S)=\left\{u \in H_{0}^{1}(Q):\left.u\right|_{S} \in \mathscr{D}(S)\right\} .
$$

Here we denote by the symbol $\left.f\right|_{S}$ the trace $\gamma_{0} f$ of $f$ to $S$ (see Appendices $\mathrm{A}$ and $\mathrm{B})$.

The space $V(Q, S)$ is nontrivial; see Proposition 3.3 of [4]. We now introduce the energy form

$$
E[u]=\int_{Q}|D u|^{2} d Q+c_{0} E_{S}\left[\left.u\right|_{S}\right]
$$

defined on the domain $V(Q, S)$. Here and in the following, $d Q$ denotes the 3-dimensional Lesbesgue measure and $E(u, v)$ denotes the corresponding bilinear form

$$
E(u, v)=\int_{Q} D u D v d Q+c_{o} E_{S}\left(\left.u\right|_{S},\left.v\right|_{S}\right)
$$

defined on $V(Q, S) \times V(Q, S)$.

As in Theorem 3.2 of [26], the following result can be proved.

Proposition 3. The form E defined in (15) is a regular Dirichlet form in $L^{2}(Q)$ and the space $V(Q, S)$ is a Hilbert space equipped with the scalar product

$$
(u, v)_{V(Q, S)}=E(u, v) .
$$

We denote by $\|u\|_{V(\mathrm{Q}, S)}$ the norm in $V(Q, S)$, associated with (17), that is

$$
\|u\|_{V(Q, S)}=\left(c_{0} E_{S}\left[\left.u\right|_{S}\right]+\int_{Q}|D u|^{2} d Q\right)^{1 / 2} .
$$

As in Propositions (3.6) and (3.1) in [4], the following result can be proved.

Proposition 4. The space $\mathscr{D}(S)$ is embedded in $B_{\beta, 0}^{2,2}, \beta=$ $d_{f} / 2$.

Proposition 5. The space $\mathscr{D}(S)$ is embedded in $B_{\alpha}^{2,2}, \alpha<1$.

As $(E, V(Q, S))$ is a closed bilinear form on $L^{2}(Q)$ with domain $V(Q, S)$ dense in $L^{2}(Q)$, there exists (see Chapter 6 Theorem 2.1 in [27]) a unique self-adjoint nonpositive operator $A$ on $L^{2}(Q)$ with domain $\mathscr{D}(A) \subseteq V(Q, S)$ dense in $L^{2}(Q)$ such that

$$
E(u, v)=-\int_{Q} A u v d Q, \quad u \in \mathscr{D}(A), v \in V(Q, S) .
$$

Moreover in Theorem 13.1 of [25] it is proved that to each closed symmetric form $E$ a family of linear operators $\left\{G_{\alpha}, \alpha>\right.$ $0\}$ can be associated with the property

$$
\begin{gathered}
E\left(G_{\alpha} u, v\right)+\alpha\left(G_{\alpha} u, v\right)=(u, v), \\
u \in L^{2}(Q), \quad v \in V(Q, S),
\end{gathered}
$$

and this family is a strongly continuous resolvent with generator $A$, which also generates a strongly continuous semigroup $\{T(t)\}_{t \geq 0}$.

For the reader's convenience, we recall here the main properties of the semigroup $\{T(t)\}_{t \geq 0}$; the reader is referred to Proposition 3.5 in [21] for the proof.

Proposition 6. Let $\{T(t)\}_{t \geq 0}$ be the semigroup generated by the operator $A$ associated with the energy form in (19). Then $\{T(t)\}_{t \geq 0}$ is an analytic contraction positive preserving semigroup in $L^{2}(Q)$.

Remark 7. It is well known that the symmetric and contraction analytic semigroup $T(t)$ uniquely determines analytic semigroups on the space $L^{p}, 1 \leq p<\infty$ (see Theorem 1.4.1 [30]) which we still denote by $T(t)$ and by $A_{p}$ its infinitesimal generator.

From Theorem 2.11 in [31], the following estimate on the decay of the heat semigroup holds.

Proposition 8. There exists a positive constant $M$ such that

$$
\|T(t)\|_{\mathscr{L}\left(L^{1} \rightarrow L^{\infty}\right)} \leq \begin{cases}M t^{-n / 2}, & \text { for every } t \in(0,1] \\ M t^{-\nu / 2}, & \text { for every } t \in[1, \infty) .\end{cases}
$$

One will consider the case $n=3$ and $v=2$; here $v$ is the spectral dimension of $S$.

From interpolation theory results, it can be proved (see Section 3.1 in [18]) that

$$
\begin{aligned}
& \|T(t)\|_{L^{2} \rightarrow L^{2 p}} \\
& \quad \leq \begin{cases}M^{(1 / 2)-(1 / 2 p)} t^{-(n / 4)(1-1 / p)}, & \text { for every } t \in(0,1] \\
M^{(1 / 2)-(1 / 2 p)} t^{-(v / 4)(1-1 / p)}, & \text { for every } t \in[1, \infty) .\end{cases}
\end{aligned}
$$


3.2. The Energy Forms $E_{S_{h}}$. By $Q$ we denote the parallelepiped as defined in Section 3 and by $S_{h}$ we denote the prefractal layer of the type $S_{h}=F_{h} \times I, h=1,2, \ldots, F_{h}$ is the prefractal approximation of $F$ at the step $h$ (see Section 2). $S_{h}$ divides $Q$ in two subsets $Q_{h}^{i}, i=1,2$.

We first construct the energy forms $E_{S_{h}}$ on the prefractal layers $S_{h}=F_{h} \times I, h \in \mathbb{N}$. By $\ell$ we denote the natural arclength coordinate on each edge of $F_{h}$ and we introduce the coordinates $x_{1}=x_{1}(\ell), x_{2}=x_{2}(\ell)$, and $y=y$ on every affine "face" $S_{h}^{(j)}$ of $S_{h}$. By $d \ell$ we denote the one-dimensional measure given by the arc-length $\ell$ and by $d \sigma$ are denote the surface measure on each face $S_{h}^{(j)}$ of $S_{h}$; that is, $d \sigma=d \ell d y$. We define $E_{S_{h}}[u]$ by setting

$$
E_{S_{h}}[u]=\sum_{j}\left(\int_{S_{h}^{(j)}}\left(\sigma_{h}^{1}\left|D_{\ell} u\right|^{2}+\sigma_{h}^{2}\left|D_{y} u\right|^{2}\right) d \sigma\right),
$$

where $\sigma_{h}^{1}$ and $\sigma_{h}^{2}$ are positive constants and $u \in H^{1}\left(S_{h}\right)$, the Sobolev space of functions on the piecewise affine set $S_{h}$ (see Appendices A and B). By Fubini theorem, we can write this functional in the form

$$
\begin{aligned}
E_{S_{h}}[u]= & \sigma_{h}^{1} \int_{I}\left(\int_{F_{h}}\left|D_{\ell} u\right|^{2} d \ell\right) d y \\
& +\sigma_{h}^{2} \int_{F_{h}}\left(\int_{I}\left|D_{y} u\right|^{2} d y\right) d \ell .
\end{aligned}
$$

We denote the corresponding bilinear form by $E_{S_{h}}(u, v)$. In the sequel we denote by the symbol $\left.f\right|_{S_{h}}$ the trace $\gamma_{0} f$ to $S_{h}$.

Consider now the space of functions $u: Q \rightarrow \mathbb{R}$ as

$$
V\left(Q, S_{h}\right)=\left\{u \in H_{0}^{1}(Q):\left.u\right|_{S_{h}} \in H_{0}^{1}\left(S_{h}\right)\right\} ;
$$

it is not trivial as it contains $\mathscr{D}(Q)$.

Consider now the energy form

$$
E^{(h)}[u]=\int_{Q}|D u|^{2} d Q+E_{S_{h}}\left[\left.u\right|_{S_{h}}\right],
$$

defined on the domain $V\left(Q, S_{h}\right)$. form

By $E^{(h)}(u, v)$ we will denote the corresponding bilinear

$$
E^{(h)}(u, v)=\int D u D v d Q+E_{S_{h}}\left(\left.u\right|_{S_{h}},\left.v\right|_{S_{h}}\right)
$$

defined on $V\left(Q, S_{h}\right) \times V\left(Q, S_{h}\right)$.

Theorem 9. The form $E^{(h)}$, defined in (26), with domain $V\left(Q, S_{h}\right)$ is a regular Dirichlet form in $L^{2}(Q)$ and the space $V\left(Q, S_{h}\right)$ is a Hilbert space equipped with the scalar product

$$
(u, v)_{V\left(Q, S_{h}\right)}=E^{(h)}(u, v) .
$$

For the proof, see Theorem 4.1 in [4].

We denote by $\|u\|_{V\left(Q, S_{h}\right)}$ the corresponding energy norm in $V\left(Q, S_{h}\right)$; that is,

$$
\|u\|_{V\left(Q, S_{h}\right)}=\left(\int_{Q}|D u|^{2} d Q+E_{S_{h}}\left[\left.u\right|_{S_{h}}\right]\right)^{1 / 2} .
$$

Proceeding as in Section 3.1 we denote by $\left\{G_{\alpha}^{h}, \alpha>0\right\}, A_{h}$, and $\left\{T_{h}(t)\right\}_{t \geq 0}$ the resolvents, the generators, and the semigroups associated to $E^{(h)}$, for every $h \in \mathbb{N}$, respectively.

As in Proposition 6, the following result can be proved.

Proposition 10. Let $\left\{T_{h}(t)\right\}_{t \geq 0}$ be the semigroup generated by the operator $A_{h}$ associated with the energy form in (27). Then $\left\{T_{h}(t)\right\}_{t \geq 0}$ is an analytic contraction positive preserving semigroup in $L^{2}(Q)$.

By proceeding as in Remark 7, one can show that for every $h \in \mathbb{N}$ the symmetric and contraction analytic semigroup $T_{h}(t)$ uniquely determines analytic semigroups on the space $L^{p}, 1<p<\infty$ (see Theorem 1.4.1 [30]) which we still denote by $T_{h}(t)$ and by $A_{p}^{h}$ its infinitesimal generator.

The following estimate on the decay of the heat semigroup holds (see e.g., [32]).

Proposition 11. There exists a positive constant $\bar{M}$ such that

$$
\left\|T_{h}(t)\right\|_{L^{1} \rightarrow L^{\infty}} \leq \begin{cases}\bar{M} t^{-n / 2}, & \text { for every } t \in(0,1] \\ \bar{M} t^{-v / 2}, & \text { for every } t \in[1, \infty),\end{cases}
$$

where $\bar{M}$ does not depend on $h$. One considers the cases $n=3$ and $v=2$; here $v$ is the Euclidean dimension of $S$.

As before by interpolation results it can be proved that

$$
\begin{aligned}
& \left\|T_{h}(t)\right\|_{L^{2} \rightarrow L^{2 p}} \\
& \quad \leq \begin{cases}\bar{M}^{(1 / 2)-(1 / 2 p)} t^{-(n / 4)(1-1 / p)}, & \text { for every } t \in(0,1] \\
\bar{M}^{(1 / 2)-(1 / 2 p)} t^{-(v / 4)(1-1 / p)}, & \text { for every } t \in[1, \infty) .\end{cases}
\end{aligned}
$$

3.3. The Convergence of Forms and Semigroups. We now recall the results proved in [21] on the convergence of the approximating energy forms $E^{(h)}$ to the fractal energy $E$. In this asymptotic behaviour, the factors $\sigma_{h}^{1}$ and $\sigma_{h}^{2}$ have a key role and can be regarded as a sort of renormalization factors of the approximating energies. These factors take into account the nonrectifiability of the curve $F$ and hence the irregularity of the surface $S$ and in particular the effect of the $D$-dimensional length intrinsic to the curve; for details, see [6]. The convergence of functional is here intended in the sense of the $M$-convergence which we define below.

3.3.1. The M-Convergence of Forms. We recall, for the sake of completeness, the definition of $M$-convergence of forms introduced by Mosco in [33].

We extend the form $E$ defined in (15) and $E^{(h)}$ defined in (26) on the whole space $L^{2}(Q)$ by defining

$$
\begin{gathered}
E[u]=+\infty \quad \text { for every } u \in \frac{L^{2}(Q)}{V(Q, S)}, \\
E^{(h)}[u]=+\infty \quad \text { for every } u \in \frac{L^{2}(Q)}{V\left(Q, S_{h}\right)} .
\end{gathered}
$$


Definition 12. A sequence of form $\left\{E^{(h)}\right\} M$-converges to a form $E$ in $L^{2}(Q)$ if

(a) for every $\left\{v_{h}\right\}$ converging weakly to $u$ in $L^{2}(Q)$

$$
\liminf _{h \rightarrow \infty} E^{(h)}\left[v_{h}\right] \geq E[u]
$$

(b) for every $u \in L^{2}(Q)$ there exists $\left\{w_{h}\right\}$ converging strongly to $u$ in $L^{2}(Q)$ such that

$$
\limsup _{h \rightarrow \infty} E^{(h)}\left[w_{h}\right] \leq E[u] .
$$

Definition 13. The sequence of forms $\left\{E^{(h)}\right\}$ is asymptotically compact in $L^{2}(Q)$ if every sequence $\left\{u_{h}\right\}$ with

$$
\liminf _{h \rightarrow \infty} E^{(h)}\left[u_{h}\right]+\int_{Q}\left|u_{h}\right|^{2} d Q<\infty
$$

has a subsequence strongly convergent in $L^{2}(Q)$.

Proposition 14. The sequence of forms (26) is asymptotically compact in $L^{2}(Q)$.

Remark 15. We point out that, as the sequence of forms (26) is asymptotically compact in $L^{2}(Q), M$-convergence is equivalent to the $\Gamma$-convergence (see Lemma 2.3.2 in [34]) and thus we can take in (a) $v_{h}$ strongly converging to $u$ in $L^{2}(Q)$.

Theorem 16. Let $\sigma_{h}^{1}=\sigma_{1} c_{0}\left(3^{d_{f}-1}\right)^{h}$ and $\sigma_{h}^{2}=\sigma_{2} c_{0}\left(3^{1-d_{f}}\right)^{h}$; then the sequence of forms $\left\{E^{(h)}\right\}$ defined in (26) M-converges in the space $L^{2}(Q)$ to the form $E$ defined in (15). The sequence of semigroups $\left\{T_{h}(t)\right\}$ associated with the form $E^{(h)}$ converges to the semigroup $T(t)$ associated with the form $E$ in the strong operator topology of $L^{2}(Q)$ uniformly on every interval $\left[0, t_{1}\right]$.

\section{Evolution Problems: Existence and Convergence of the Solutions}

In this Section we recall the results on existence and uniqueness of the solution of the abstract problems $(P)$ and $\left(P_{h}\right)$ (see below) and the asymptotic behaviour of the solutions of the abstract problems. In Section 5 we will show that the solutions of the abstract problems solve $(\bar{P})$ in both cases. We refer the reader to [18].

We consider the abstract Cauchy problems as

$$
(P)\left\{\begin{array}{l}
\frac{d u(t)}{d t}=A u(t)+J(u(t)), \quad 0 \leq t \leq T \\
u(0)=\phi
\end{array}\right.
$$

and for every $h \in \mathbb{N}$

$$
\left(P_{h}\right)\left\{\begin{array}{l}
\frac{d u_{h}(t)}{d t}=A_{h} u_{h}(t)+J\left(u_{h}(t)\right), \quad 0 \leq t \leq T \\
u_{h}(0)=\phi_{h},
\end{array}\right.
$$

where $A: \mathscr{D}(A) \subset L^{2}(Q) \rightarrow L^{2}(Q)$ and $A_{h}: \mathscr{D}\left(A_{h}\right) \subset$ $L^{2}(Q) \rightarrow L^{2}(Q)$ are the generators associated, respectively, to the energy form $E$ and the energy forms $E^{(h)}$ introduced in (15) and (26), T is a fixed positive real number, and $\phi$ and $\phi_{h}$ are given functions in $L^{2}(Q)$. We assume that $J$ is a mapping from $L^{2 p}(Q) \rightarrow L^{2}(Q), p>1$ locally Lipschitz, that is, Lipschitz on bounded sets in $L^{2 p}(Q)$; we let $l(r)$ denote the Lipschitz constant of $J$ :

$$
\|J(u)-J(v)\|_{L^{2}(\mathrm{Q})} \leq l(r)\|u-v\|_{L^{2 p}(\mathrm{Q})},
$$

where $\|u\|_{L^{2 p}(Q)} \leq r,\|v\|_{L^{2 p}(Q)} \leq r$. We also assume that $J(0)=$ 0 . This assumption is not necessary in all that follows, but it simplifies the calculations (see [11]). In order to prove the local existence theorem, we make the following assumptions on the growth of $l(r)$ when $r \rightarrow \infty$.

We set for brevity $a:=(n / 4)(1-(1 / p))$; we note that $0<$ $a<1$, for $n \leq 4$, and $p>1$.

(i) There exists $0<b<a$ such that $l(r)=$ $\mathcal{O}\left(r^{(1-a) / b}\right), r \rightarrow \infty$.

(ii) Consider

$$
\int_{\tau}^{+\infty} l(r) r^{-1 / a} d r<\infty
$$

for every $\tau>0$.

We note that (ii) implies (i) for all $0<b<a$ since $l(r)$ is nondecreasing and

$$
\int_{r}^{2 r} l(s) s^{-1 / a} d s \geq r l(r)(2 r)^{-1 / a} .
$$

Thus $l(r) r^{1-(1 / a)}$ is bounded as $r \rightarrow \infty$ which implies (i) for $0<b<a$.

In Theorem 5.1 of [18], the following local existence theorem has been proved.

Theorem 17. Let condition (i) hold. Let $K>0$ be sufficiently small if $\phi \in L^{2}(Q)$ and

$$
\limsup _{t \rightarrow 0}\left\|t^{b} T(t) \phi\right\|_{L^{2 p}}<K .
$$

There is $a T>0$ and $a$

$$
u \in C\left([0, T] ; L^{2}(Q)\right)
$$

with $u(0)=\phi$ satisfying

(1) $u \in C\left((0, T] ; L^{2 p}(Q)\right)$, and $\left\|t^{b} u(t)\right\|_{L^{2 p}}<2 K$;

(2) for every $t \in[0, T]$,

$$
u(t)=T(t) \phi+\int_{0}^{t} T(t-s) J(u(s)) d s
$$

with the integral being both an $L^{2}$-valued and $L^{2 p}$. valued Bochner integral; 
(3) if $v:\left(0, T_{1}\right] \rightarrow L^{2 p}$ is strongly measurable with $T_{1} \leq$ $T,\left\|t^{b} v(t)\right\|_{L^{2 p}} \leq 2 K$ and also satisfies (43), then $u(t)=$ $v(t)$, for every $t \in\left(0, T_{1}\right]$.

Let condition (ii) hold; there exist $a T>0$ and a unique $u(t) \in C\left([0, T] ; L^{2}(Q)\right)$ with $u(0)=\phi$ satisfying

(1) $u \in C\left((0, T] ; L^{2 p}(Q)\right)$

$$
\limsup _{t \rightarrow 0}\left\|t^{a} u(t)\right\|_{L^{2 p}}<\infty ;
$$

(2) for every $t \in[0, T], u(t)$ satisfies (43) with the integral being both an $L^{2}$-valued and $L^{2 p}$-valued Bochner integral;

(3) if $v:\left(0, T_{1}\right] \rightarrow L^{2 p}$ is strongly measurable with $T_{1} \leq T,\left\|t^{a} v(t)\right\|_{L^{2 p}}$ bounded and also satisfies (43), then $u(t)=v(t)$, for every $t \in\left(0, T_{1}\right]$.

The claim of the Theorem is proved by a contraction mapping argument on suitable spaces of continuous functions with values in Banach space.

By exploiting the analyticity of the semigroup $T(t)$ both on $L^{2}(Q)$ and $L^{2 p}(Q)$, the following regularity result for the maximal solution holds (see Theorem 5.3 [18]).

Theorem 18. Under the assumptions of Theorem 17, one has that the solution $u(t)$ can be continuously extended to a maximal interval $\left(0, T_{\phi}\right)$ as a solution of $(43)$, until $\|u(t)\|_{L^{2 p}(\mathrm{Q})} \rightarrow$ $\infty$ as $t \rightarrow T_{\phi}$, and it is a classical solution; that is,

$$
u \in C^{1}\left(\left(0, T_{\phi}\right) ; L^{2}(Q)\right) \cap C\left(\left(0, T_{\phi}\right) ; \mathscr{D}(A)\right)
$$

and satisfies

$$
\frac{d u(t)}{d t}=A u(t)+J(u), \quad \text { for every } t \in\left(0, T_{\phi}\right) .
$$

For every fixed $h \in \mathbb{N}$, the claims of Theorems 17 and 18 hold for problem $\left(P_{h}\right)$ with the obvious changes.

We now recall the convergence results of the sequence of the approximating solutions $\left\{u_{h}\right\}$ when h goes to infinity (see Theorem 6.2 in [18]).

Theorem 19. Let $u$ and $u_{h}$ be the mild solutions of problems $(P)$ and $\left(P_{h}\right) ;$ let $\sigma_{h}^{1}$ and $\sigma_{h}^{2}$ be as in Theorem 16. In the notations and assumptions of Theorem 17, one has the following;

(a) let assumption (i) hold; let $\phi_{h}$ and $\phi$ belong to $L^{q}(Q)$ with $q=2 p n /(n+4 p b)$ and $\phi_{h} \rightarrow \phi$ in $L^{q}(Q)$; then

$$
\begin{gathered}
u_{h} \rightarrow u \quad \text { in } C\left([0, T] ; L^{2}(Q)\right), \\
\sup _{t \in[0, T]}\left\|t^{b}\left[u(t)-u_{h}(t)\right]\right\|_{L^{2 p}(Q)} \longrightarrow 0 ;
\end{gathered}
$$

(b) if assumption (ii) holds and $\phi_{h} \rightarrow \phi$ in $L^{2}(Q)$, then

$$
\begin{gathered}
u_{h} \rightarrow u \quad \text { in } C\left([0, T] ; L^{2}(Q)\right) \\
\sup _{t \in[0, T]}\left\|t^{a}\left[u(t)-u_{h}(t)\right]\right\|_{L^{2 P}(Q)} \longrightarrow 0
\end{gathered}
$$

with $a=n / 4(1-1 / p)$.

\section{Strong Formulation of the Transmission Problems}

5.1. The Fractal Layer

Theorem 20. Let $u$ be the solution of problem $(P)$. Then one has, for every fixed $t \in(0, T]$,

$$
\begin{gathered}
u_{t}(t, P)-\Delta u(t, P)=J(u(t, P)) \\
\text { for a.e. } P \in Q_{i} i=1,2, \\
\frac{\partial u^{i}}{\partial n_{i}} \in\left(B_{\beta, 0}^{2,2}(S)\right)^{\prime}, \quad \beta=\frac{d_{f}}{2}, i=1,2, \\
-c_{0}\left\langle\left.\Delta_{S} u\right|_{S}, z\right\rangle_{(\mathscr{D}(S))^{\prime} \mathscr{D}(S)} \\
=\left\langle\left[\frac{\partial u}{\partial n}\right], z\right\rangle_{(\mathscr{D}(S))^{\prime} \mathscr{D}(S)}, \quad \text { for every } z \in \mathscr{D}(S), \\
u(t, P)=0 \quad \text { for } P \in \partial Q, \\
u^{1}=u^{2} \quad \text { in } B_{d_{f} / 2}^{2,2}(S), \\
u(0, P)=\phi(P) \quad \text { in } L^{2}(Q),
\end{gathered}
$$

where $u^{i}$ is the restriction of $u$ to $Q_{i}, \partial u^{i} / \partial n_{i}, i=1,2$ is the inward "normal derivative," to be defined in a suitable sense, $[\partial u / \partial n]=\left(\partial u^{1} / \partial n_{1}\right)+\left(\partial u^{2} / \partial n_{2}\right)$ is the jump of the normal derivative, and $\Delta_{S}$ is the fractal Laplacian. Moreover $\partial u^{i} / \partial n_{i} \epsilon$ $C\left((0, T] ;\left(B_{\beta, 0}^{2,2}(S)\right)^{\prime}\right)$.

Proof. Let $\varphi(P)$ be an arbitrary function in $V(Q, S)$ such that $\left.\varphi(P)\right|_{Q_{i}} \in \mathscr{D}\left(Q_{i}\right)$; by multiplying for $\varphi(36)$ in $(P)$ and integrating over $Q$ we have

$$
\begin{aligned}
\int_{Q} u_{t}(t, P) \varphi(P) d Q= & \int_{Q} A u(t, P) \varphi(P) d Q \\
& +\int_{Q} J(u(t, P)) \varphi(P) d Q .
\end{aligned}
$$

From (19) and taking into account that $\varphi \in \mathscr{D}\left(Q_{i}\right)$, we have

$$
\begin{aligned}
\int_{Q_{i}} u_{t}(t, P) \varphi(P) d Q= & -\int_{Q_{i}} D u(t, P) D \varphi(P) d Q \\
& +\int_{Q_{i}} J(u(t, P)) \varphi(P) d Q \\
= & \int_{Q_{i}} \Delta u(t, P) \varphi(P) d Q \\
& +\int_{Q_{i}} J(u(t, P)) \varphi(P) d Q .
\end{aligned}
$$

From the arbitrariness of $\varphi$, we have that, for fixed $t \in(0, T]$,

$$
u_{t}(t, P)=\Delta u(t, P)+J(u(t, P)) \text { holds in } \mathscr{D}^{\prime}\left(Q_{i}\right)
$$

From the density of $\mathscr{D}\left(Q_{i}\right)$ in $L^{2}\left(Q_{i}\right)$ and since $J(u(t, \cdot)) \in$ $L^{2}(Q)$, we obtain the first assertion in (49). From this equality, 
we obtain $\Delta u(t, P)=u_{t}(t, P)-J(u(t, P))$ and since the right-hand side belongs to $C\left((0, T] ; L^{2}\left(Q_{i}\right)\right)$ we deduce that $\Delta u(t, P) \in C\left((0, T] ; L^{2}\left(Q_{i}\right)\right)$; hence $u(t, \cdot) \in C\left((0, T] ; V\left(Q_{i}\right)\right)$, where

$$
V\left(Q_{i}\right)=\left\{u \in H_{0}^{1}(Q) ; \Delta u^{i} \in L^{2}\left(Q_{i}\right)\right\} ;
$$

here the Laplacian is intended in the distributional sense. By proceeding as in (3.26) of [4], we prove that, for every fixed $t$, the normal derivative $\partial u^{i} / \partial n_{i}$ is in the dual $\left(B_{\beta, 0}^{2,2}(S)\right)^{\prime}$ of the space $\left(B_{\beta, 0}^{2,2}(S)\right)$, where $\beta=d_{f} / 2$ and

$$
\begin{aligned}
\left\langle\frac{\partial u^{i}}{\partial n_{i}},\left.v\right|_{S}\right\rangle_{\left(\left(B_{\beta, 0}^{2,2}(S)\right)^{\prime}\right),\left(\left(B_{\beta, 0}^{2,2}(S)\right)\right)} & =\int_{Q_{i}} D u(t, P) D v(P) d Q \\
& +\int_{Q_{i}} v(P) \Delta u^{i}(t, P) d Q
\end{aligned}
$$

for every $t \in(0, T]$ and every $v \in H_{0}^{1}(Q)$. By proceeding as in Section 6.1 of [21], we can prove that $\partial u^{i} / \partial n_{i} \in$ $C\left((0, T] ;\left(B_{\beta, 0}^{2,2}(S)\right)^{\prime}\right)$.

From Proposition 4 and proceeding as in Section 6 of [3], it can be proved that the transmission condition

$$
-\left.c_{0} \Delta_{S} u\right|_{S}=\left[\frac{\partial u}{\partial n}\right] \text { holds in }(\mathscr{D}(S))^{\prime} .
$$

That is, for every $t \in(0, T]$,

$$
-c_{0}\left\langle\left.\Delta_{S} u\right|_{S}, z\right\rangle_{(\mathscr{D}(S))^{\prime} \mathscr{D}(S)}=\left\langle\left[\frac{\partial u}{\partial n}\right], z\right\rangle_{(\mathscr{D}(S))^{\prime} \mathscr{D}(S)} .
$$

As a consequence of Theorem 20, the solution of problem $(P)$ is the solution of the following transmission problem. For every $t \in(0, T]$,

(j)

$$
u_{t}^{i}-\Delta u^{i}=J\left(u^{i}\right) \quad \text { in } L^{2}\left(Q_{i}\right), i=1,2,
$$

(ji)

$$
-c_{0} \Delta_{S} u=\left[\frac{\partial u}{\partial n}\right] \quad \text { in }(\mathscr{D}(S))^{\prime},
$$

(jjj)

$$
u=0 \quad \text { in } H^{1 / 2}(\partial Q),
$$

(jv)

$$
u^{1}=u^{2} \quad \text { in } B_{d_{f} / 2}^{2,2}(S)
$$

(v)

$$
u=0 \quad \text { in } B_{\left(d_{f}-1\right) / 2}^{2,2}(\partial S)
$$

Remark 21. Actually from Proposition 6, one deduces that equalities (jv) and (v), respectively, hold in $B_{\alpha}^{2,2}(S)$ and in $B_{\alpha-(1 / 2)}^{2,2}(\partial S)$ with $\alpha<1$.

\subsection{The Prefractal Layer}

Theorem 22. Let $u_{h}$ be the solution of problem $\left(P_{h}\right)$. Then one has, for every fixed $t \in(0, T]$,

$$
\begin{gathered}
\left(u_{h}\right)_{t}(t, P)-\Delta u_{h}(t, P) \\
=J\left(u_{h}(t, P)\right) \quad \text { for } P \in Q_{h}^{i}, \text { a.e. } i=1,2, \\
\frac{\partial u_{h}^{i}}{\partial n_{i}} \in L^{2}\left(S_{h}\right), \quad i=1,2, \\
-\left.\Delta_{S_{h}} u\right|_{S_{h}}=\left[\frac{\partial u_{h}}{\partial n}\right], \quad \text { in } L^{2}\left(S_{h}\right), \\
u(t, P)=0 \quad \text { for } P \in \partial Q, \\
u_{h}^{1}=u_{h}^{2} \quad \text { in } H_{0}^{1}\left(S_{h}\right), \\
u_{h}(0, P)=\phi(P) \quad \text { in } L^{2}(Q),
\end{gathered}
$$

where $u_{h}^{i}$ is the restriction of $u_{h}$ to $Q_{h}^{i},\left[\partial u_{h} / \partial n\right]=\left(\partial u_{h}^{1} / \partial n_{1}\right)+$ $\left(\partial u_{h}^{2} / \partial n_{2}\right)$ is the jump of the normal derivatives across $S_{h}, n_{i}$, $i=1,2$, is the inward normal vector, and $\Delta_{S_{h}}=\sigma_{h}^{1} D_{\ell}^{2}+\sigma_{h}^{2} D_{y}^{2}$ is the piecewise tangential Laplacian associated to the Dirichlet form $E_{S_{h}}$. Moreover $\partial u_{h}^{i} / \partial n_{i} \in C\left((0, T] ; L^{2}\left(S_{h}\right)\right)$.

Proof. The first equality in (62) easily follows by proceeding as in Theorem 20. From this, it follows that, for every $t \in[0, T]$,

$$
u_{h}(t, \cdot) \in V\left(Q_{h}^{i}\right)=\left\{u \in H_{0}^{1}(Q) ; \Delta u_{h}^{i} \in L^{2}\left(Q_{h}^{i}\right)\right\} .
$$

For every fixed $t \in(0, T]$, let $u_{h}^{i}$ denote the restriction of the solution $u_{h}$ to $Q_{h}^{i}$. By usual duality arguments (see Appendix 4 in [35]), the normal derivatives $\partial u_{h}^{i} / \partial n_{i}, i=1,2$ belong to the dual space of $H_{0,0}^{1 / 2}\left(S_{h}\right)$. By proceeding as in Section 6.2 of [21], it is possible to prove that $\partial u_{h}^{i} / \partial n_{i} \in$ $C\left((0, T] ;\left(H_{0,0}^{1 / 2}\left(S_{h}\right)\right)^{\prime}\right)$.

Then, by the Green formula for Lipschitz domains, one can prove that

$$
\begin{aligned}
&\left\langle-\left.\Delta_{S_{h}} u\right|_{S_{h}}, z\right\rangle_{\left(H_{0,0}^{1 / 2}\left(S_{h}\right)\right)^{\prime} H_{0,0}^{1 / 2}\left(S_{h}\right)} \\
&=\left\langle\left[\frac{\partial u_{h}}{\partial n}\right], z\right\rangle_{\left(H_{0,0}^{1 / 2}\left(S_{h}\right)\right)^{\prime} H_{0,0}^{1 / 2}\left(S_{h}\right)} .
\end{aligned}
$$

That is, the transmission condition

$$
-\left.\Delta_{S_{h}} u\right|_{S_{h}}=\left[\frac{\partial u_{h}}{\partial n}\right]
$$

holds in the dual of $H_{0,0}^{1 / 2}\left(S_{h}\right)$ (see Proposition 2.2 in [5] for details). In order to prove that $\partial u_{h}^{i} / \partial n_{i} \in L^{2}\left(S_{h}\right)$, we proceed as in Section 4.2 of [4]. Let us consider, for each fixed 
$t \in(0, T]$, the weak solutions $w_{h}^{i}$ and $\widehat{w}_{h}^{i}$ in $H^{1}\left(Q_{h}^{i}\right)$ of the following auxiliary problems:

$$
\begin{gathered}
\Delta \widehat{w}_{h}^{i}=0 \quad \text { in } Q_{h}^{i}, \\
\widehat{w}_{h}^{i}=u_{h} \quad \text { on } \partial Q_{h}^{i}, \\
-\Delta w_{h}^{i}=-\left(u_{h}^{i}\right)_{t}+J\left(u_{h}^{i}\right) \quad \text { in } Q_{h}^{i}, \\
w_{h}^{i}=0 \quad \text { on } \partial Q_{h}^{i},
\end{gathered}
$$

The regularity of $u_{h}^{i}$ follows from the regularity of $w_{h}^{i}$ and $\widehat{w}_{h}^{i}$ since

$$
u_{h}^{i}=w_{h}^{i}+\widehat{w}_{h}^{i} .
$$

From a regularity result of Jerison and Kenig (see Theorems 2 and 3 of [36]), we deduce that

$$
\frac{\partial \widehat{w}_{h}^{i}}{\partial n_{i}} \in L^{2}\left(S_{h}\right)
$$

and $\left\|\partial \widehat{w}_{h}^{i} / \partial n_{i}\right\| \leq C(h)\left\|u_{h}\right\|_{V\left(Q ; S_{h}\right)} \leq C(h)\left\|u_{h}\right\|_{D\left(A_{h}\right)}$.

As to the solution $w_{h}^{i}$ of (67), we preliminary observe that the right-hand side in the first equation of (67) belongs to $L^{2}\left(Q_{h}^{i}\right)$. From Proposition 4.5 in [4], it follows that

$$
w_{h}^{i} \in H^{s_{i}}\left(Q_{h}^{i}\right), \quad i=1,2,
$$

where $1<s_{1}<8 / 5$ and $1<s_{2}<7 / 4$; hence

$$
\begin{array}{r}
D^{\alpha} w_{h}^{1} \in H^{(3 / 5)-\epsilon}\left(Q_{h}^{1}\right), \quad D^{\alpha} w_{h}^{2} \in H^{(3 / 4)-\epsilon}\left(Q_{h}^{2}\right), \\
|\alpha|=1
\end{array}
$$

for every $\epsilon>0$; then by trace results (see Proposition A.1), we obtain, for $i=1,2$,

$$
\frac{\partial w_{h}^{i}}{\partial n_{i}} \in L^{2}\left(S_{h}\right)
$$

and $\left\|\partial w_{h}^{i} / \partial n_{i}\right\|_{L^{2}\left(S_{h}\right)} \leq c(h)\left\|-\left(u_{h}^{i}\right)_{t}+J\left(u_{h}^{i}\right)\right\|_{L^{2}(Q)}$. It follows from (67), (68), and (69) that $\partial u_{h}^{i} / \partial n_{i} \in L^{2}\left(S_{h}\right), i=1,2$; hence the jump belongs to $L^{2}\left(S_{h}\right)$. As $H_{0,0}^{1 / 2}\left(S_{h}\right)$ is dense in $L^{2}\left(S_{h}\right)$ (see e.g., [37]), we deduce that the transmission condition (64) actually holds in the $L^{2}$-sense and in particular $\Delta_{S_{h}} u_{h} \in$ $L^{2}\left(S_{h}\right)$. The proof that $\partial u_{h}^{i} / \partial n_{i} \in C\left((0, T] ; L^{2}\left(S_{h}\right)\right)$ easily follows from (69), (72), and the fact that $u_{h}, A_{h} u_{h}, J\left(u_{h}\right)$, and $\left(u_{h}^{i}\right)_{t}$ belong to $C\left((0, T] ; L^{2}(Q)\right)$.

From Theorem 22, it follows that the solution of problem $\left(P_{h}\right)$ is the solution of the following transmission problem. For every $t \in(0, T]$,

(j)

$$
u_{t}^{i}=\Delta u^{i}+J\left(u^{i}\right) \quad \text { in } L^{2}\left(Q_{h}^{i}\right), i=1,2,
$$

(ji)

$$
-\Delta_{S_{h}} u=\left[\frac{\partial u}{\partial n}\right] \quad \text { in } L^{2}\left(S_{h}\right)
$$

(jjj)

$$
u=0 \quad \text { in } H^{1 / 2}(\partial Q)
$$

(jv)

$$
u^{1}=u^{2} \quad \text { in } H^{1}\left(S_{h}\right)
$$

(v)

$$
u=0 \quad \text { in } H^{1 / 2}\left(\partial S_{h}\right)
$$

\section{Convergence Results}

Now we are interested in the behavior of the sequence $\left\{u_{h}\right\}$ when $h$ goes to $\infty$.

Theorem 23. Let $u$ and $u_{h}$ be the solutions of problems $(P)$ and $\left(P_{h}\right)$ according to Theorem 19. Let $\sigma_{h}^{1}$ and $\sigma_{h}^{2}$ be as in Theorem 16. For every fixed positive $\epsilon$, one has

(i) $J\left(u_{h}\right)$ converges to $J(u)$ in $L^{2}([\epsilon, T] \times Q)$;

(ii) $\left\{d u_{h} / d t\right\}$ weakly converges to $d u / d t$ in $L^{2}([\epsilon, T] \times Q)$;

(iii) $\left\{A_{h} u_{h}\right\}$ weakly converges to $A u$ in $L^{2}([\epsilon, T] \times Q)$;

(iv) $\left\{u_{h}\right\}$ converges to $u$ in $L^{2}\left([\epsilon, T] ; H_{0}^{1}(Q)\right)$.

Proof. We prove condition (i), that is,

$$
\left\|j(u(t, P))-J\left(u_{h}(t, P)\right)\right\|_{L^{2}((\epsilon, T] \times Q)} \longrightarrow 0 .
$$

From (38), we have

$$
\begin{aligned}
\int_{\epsilon}^{T}\left\|J(u(t, P))-J\left(u_{h}(t, P)\right)\right\|_{L^{2}(Q)}^{2} d t \\
\quad \leq \int_{\epsilon}^{T} l^{2}(R)\left\|u(t)-u_{h}(t)\right\|_{L^{2 p}(Q)}^{2} d t \\
\quad \leq(T-\epsilon) \sup _{t \in[\epsilon, T]} l^{2}(R)\left\|u(t)-u_{h}(t)\right\|_{L^{2 p}(Q)}^{2} .
\end{aligned}
$$

From Theorem 19 (a), we have

$$
\sup _{t \in[0, T]}\left\|t^{b}\left[u(t)-u_{h}(t)\right]\right\|_{L^{2 p}(Q)} \longrightarrow 0 .
$$

And hence, for every fixed $\epsilon>0$,

$$
\sup _{t \in[\epsilon, T]}\left\|\left[u(t)-u_{h}(t)\right]\right\|_{L^{2 p}(Q)} \longrightarrow 0 .
$$

This concludes the proof of condition (i).

We now prove condition (ii). From the local Lipschitz continuity of $J(u)$ and the Hölder continuity of $u_{h}(t)$ in $(\epsilon, T)$ into $L^{2 p}$, one can prove that $\left\|J\left(u_{h}\right)\right\|_{C^{\theta}\left([\epsilon, T] ; L^{2}(Q)\right)}$ is bounded by a constant which does not depend on $h$; actually the constants depend only on the constants of the semigroups which in turn 
do not depend on $h$. From this, together with Theorem 18, we have that there exists a constant $c$ independent of $h$ such that

$$
\begin{gathered}
\left\|u_{h}\right\|_{C^{1}\left([\epsilon, T) ; L^{2}(Q)\right)}+\left\|u_{h}\right\|_{C^{0}([\epsilon, T] ; D(A))} \\
\leq c\left\|J\left(u_{h}\right)\right\|_{C^{\theta}\left([\epsilon, T] ; L^{2}(Q)\right)} \\
\leq c l(r)\left\|u_{h}\right\|_{C^{\theta}\left([\epsilon, T] ; L^{2 p}(Q)\right)} .
\end{gathered}
$$

Thus in particular it holds $\sup _{t \in[\epsilon, T]}\left\|\left(u_{h}\right)_{t}\right\|_{L^{2}(Q)} \leq c$; thus, for every fixed $t \in[\epsilon, T],\left\|\left(u_{h}\right)_{t}\right\|_{L^{2}(\mathrm{Q})} \leq c$.

From (82), it follows that for each $h, d u_{h} / d t$ belongs to $L^{2}([\epsilon, T] \times Q)$ and $\left\|d u_{h} / d t\right\|_{L^{2}([\epsilon, T] \times Q)}^{2} \leq c$.

From the boundedness of the sequence $\left\{d u_{h} / d t\right\}$ in $L^{2}([\epsilon, T] \times Q)$, it follows that there exists a subsequence, which we denote with $\left\{d u_{h} / d t\right\}$ and a function $v \in L^{2}([\epsilon, T] \times Q)$ such that $\left\{d u_{h} / d t\right\}$ weakly converges to $v$ in $L^{2}([\epsilon, T] \times Q)$ as $h$ goes to $\infty$.

In order to prove (ii), it is enough to prove that $v=d u / d t$.

Since $C^{1}([\epsilon, T] \times Q)$ is dense in $L^{2}([\epsilon, T] \times Q)$, for every $\varphi \in C^{1}([\epsilon, T] \times Q)$, we have

$$
\begin{array}{r}
\lim _{n \rightarrow \infty} \int_{Q} \int_{\epsilon}^{T} \frac{d u_{n}}{d t}(t, P) \varphi(t, P) d t d Q \\
=\int_{Q} \int_{\epsilon}^{T} v(t, P) \varphi(t, P) d t d Q .
\end{array}
$$

Integrating by parts the left-hand side, we get

$$
\begin{aligned}
\int_{Q} \int_{\epsilon}^{T} \frac{d u_{n}}{d t}(t, P) \varphi(t, P) d t d Q & \\
= & \int_{Q}\left[u_{n}(T, P) \varphi(T, P)-u_{n}(0, P) \varphi(0, P)\right] d Q \\
& -\int_{Q} \int_{\epsilon}^{T} u_{n}(t, P) \frac{d \varphi}{d t}(t, P) d t d Q .
\end{aligned}
$$

From (47) or (48), we have

$$
\begin{aligned}
& \lim _{n \rightarrow \infty} \int_{Q} \int_{\epsilon}^{T} \frac{d u_{n}}{d t}(t, P) \varphi(t, P) d t d Q \\
& =\int_{Q}[u(T, P) \varphi(T, P)-u(0, P) \varphi(0, P)] d Q \\
& -\int_{Q} \int_{\epsilon}^{T} u(t, P) \frac{d \varphi}{d t}(t, P) d t d Q \\
& \quad=\int_{Q} \int_{\epsilon}^{T} \frac{d u}{d t}(t, P) \varphi(t, P) d t d Q .
\end{aligned}
$$

From the uniqueness of weak limit, we get $v=d u / d t$ a.e.. From the convergence of the sequence $\left\{u_{h}\right\}$ to $u$ in $L^{2}([\epsilon, T] \times$ $Q)$ and the weak convergence of the subsequence $\left\{d u_{n} / d t\right\}$ to $d u / d t$ in $L^{2}([\epsilon, T] \times Q)$, we deduce that the whole sequence $\left\{d u_{h} / d t\right\}$ weakly converges to $d u / d t$ in $L^{2}([\epsilon, T] \times Q)$.

We now prove condition (iii). It is an easy consequence of (i) and (ii). In fact $A_{h} u_{h}=\left(d u_{h} / d t\right)-J\left(u_{h}\right)$; taking the weak limit in $L^{2}([\epsilon, T] \times Q)$, we get the thesis.
We now prove condition (iv). From (i), (iii), and the property of the scalar product in $L^{2}([\epsilon, T] \times Q)$, we get that

$$
\lim _{h \rightarrow \infty}\left(A_{h} u_{h}, u_{h}\right)_{L^{2}([\epsilon, T] \times Q)}=(A u, u)_{L^{2}([\epsilon, T] \times Q)} .
$$

That is,

$$
\begin{array}{r}
\lim _{h \rightarrow \infty} \int_{\epsilon}^{T} d t \int_{Q} A_{h} u_{h}(t, P) u_{h}(t, P) d Q \\
=\int_{\epsilon}^{T} d t \int_{Q} A u(t, P) u(t, P) d Q
\end{array}
$$

From the relation between a Dirichlet form and the associated generator, it follows that

$$
\lim _{h \rightarrow \infty} \int_{\epsilon}^{T} E^{(h)}\left[u_{h}\right] d t=\int_{\epsilon}^{T} E[u] d t
$$

There exists a constant $c$ such that

$$
\begin{array}{r}
\int_{\epsilon}^{T} \int_{Q}\left|D u_{h}(t, P)\right|^{2} d Q d t+\int_{\epsilon}^{T} E_{S_{h}}\left[u_{h}\right] d t \leq c, \\
\text { for every } h \in \mathbb{N} .
\end{array}
$$

Hence

$$
\left\|D u_{h}\right\|_{L^{2}([\epsilon, T] \times Q)} \leq c .
$$

There exists a subsequence $D u_{n}$ weakly converging to $w$ in $L^{2}([\epsilon, T] \times Q)^{3}$. We now prove that

$$
\begin{aligned}
\int_{\epsilon}^{T} \int_{Q} D u_{h}(t, P) D u d Q d t \\
\quad \longrightarrow \int_{\epsilon}^{T} \int_{Q}|D u(t, P)|^{2} d Q d t .
\end{aligned}
$$

From Theorem 19, it follows in particular that $u_{n}$ converges to $u$ in $L^{2}([\epsilon, T] \times Q)$; hence $w=D u$ and $u_{h} \rightarrow u$ in $L^{2}\left([\epsilon, T] ; H_{0}^{1}(Q)\right.$; in particular (91) holds. We now prove assertion (iv) as

$$
\begin{aligned}
0 \leq & \int_{\epsilon}^{T}\left\|u_{h}(t)-u(t)\right\|_{H_{0}^{1}(Q)}^{2} d t \\
= & \int_{\epsilon}^{T} d t \int_{Q}\left|D u_{h}(t, P)-D u(t, P)\right|^{2} d Q \\
= & \int_{\epsilon}^{T} d t \int_{Q}\left(\left|D u_{h}(t, P)\right|^{2}+|D u(t, P)|^{2}\right. \\
= & \left.\quad-2 D u_{h}(t, P) D u(t, P)\right) d Q \\
& \left.\quad+\int_{\epsilon}^{T} d t E_{Q}^{(h)}\left[u_{h}\right]-E_{S_{h}}\left[u_{h}\right]\right) d t
\end{aligned}
$$


Taking the upper limit as $h \rightarrow \infty$, we have

$$
\begin{aligned}
& 0 \leq \lim \sup \left\|u_{h}(t)-u(t)\right\|_{L^{2}\left([\epsilon, T] ; H_{0}^{1}(Q)\right)}^{2} \\
& \leq \int_{\epsilon}^{T} E[u] d t \\
& \quad-\liminf _{h \rightarrow \infty} \int_{\epsilon}^{T} E_{S_{h}}\left[u_{h}\right] d t \\
& \quad+\int_{\epsilon}^{T} d t \int_{Q}|D u(t, P)|^{2} d Q \\
& \quad-2 \int_{\epsilon}^{T} d t \int_{Q}|D u(t, P)|^{2} d Q \\
& \leq c_{0} \int_{\epsilon}^{T} E_{S}[u] d t-\liminf _{h \rightarrow \infty} \int_{\epsilon}^{T} E_{S_{h}}\left[u_{h}\right] d t \\
& \leq 0, \quad
\end{aligned}
$$

where the last inequality follows from (4.9) in [21]. Hence the sequence $\left\{u_{h}\right\}$ converges to $u$ in $L^{2}\left([\epsilon, T] ; H_{0}^{1}(Q)\right)$ and therefore $\left\{D u_{h}\right\}$ converges to $D u$ in $L^{2}\left([\epsilon, T] ;\left(L^{2}(Q)\right)^{3}\right)$.

Proposition 24. Let $u$ and $u_{h}$ be the solutions of problems $(P)$ and $\left(P_{h}\right)$, respectively. Then $u$ and $u_{h} \in H^{1}([\epsilon, T] \times Q)$.

Proof. We prove the thesis for $u$. From Theorem 18, it follows that $u \in C([\epsilon, T] ; \mathscr{D}(A))$ and $(d u / d t) \in C\left([\epsilon, T] ; L^{2}(Q)\right)$. Since $\mathscr{D}(A) \subset V(Q, S) \subset H_{0}^{1}(Q)$, we obtain $u \in$ $C\left([\epsilon, T] ; H_{0}^{1}(Q)\right)$; hence $D u \in C\left([\epsilon, T] ;\left(L^{2}(Q)\right)^{3}\right)$. The thesis follows as $C\left([\epsilon, T] ; L^{2}(Q)\right) \subset L^{2}([\epsilon, T] \times Q)$. The result for $u_{h}$ can be proved analogously.

\section{Appendices}

Here we recall some definitions of functional spaces and trace results.

\section{A. Sobolev Spaces}

Let $Q$ be a polyhedral domain; just to fix the ideas, the parallelepiped is as in Section 2. For every integer $h \geq 1$, let $S_{h}$ be the prefractal surface approximating the Koch-type surface $S$ and let us denote every affine "face" of $S_{h}$ by $S_{h}^{(j)} ; S_{h}$ divides $Q$ into two subsets $Q_{h}^{1}$ and $Q_{h}^{2}$.

By $L^{p}(\cdot), p>1$ we denote the Lebesgue space with respect to the Lebesgue measure on subsets of $\mathbb{R}^{3}$, which will be left to the context whenever that does not create ambiguity. Let $\mathscr{T}$ be a closed set of $\mathbb{R}^{3}$; by $C(\mathscr{T})$ we denote the space of continuous functions on $\mathscr{T}$; by $C_{0}(\mathscr{T})$ we denote the space of continuous functions vanishing on $\partial \mathscr{T}$. Let $\mathscr{G}$ be an open set of $\mathbb{R}^{3}$; by $H^{1}(\mathscr{G})$ we denote the usual Sobolev spaces (see Necas [38]); $H_{0}^{1}(\mathscr{G})$ is the closure of $\mathscr{D}(\mathscr{G})$ (the smooth functions with compact support on $\mathscr{G}$ ), with respect to the $\|\cdot\|_{H^{1}}$-norm. In the following, we will make use of trace spaces on boundaries of polyhedral domains of $\mathbb{R}^{3}$.

By $H_{0}^{1}\left(S_{h}\right)$ we denote the closure in $H^{1}\left(S_{h}\right)$ of the set

$$
\begin{aligned}
& \left\{\left.v\right|_{\partial Q_{h}^{2}}: v \in C^{\infty}\left(Q_{h}^{2}\right) ;\right. \\
& \left.\quad v \text { vanishes in a neighborhood of } S_{h}\right\} .
\end{aligned}
$$

By $H^{r}\left(S_{h}\right), 0<r \leq 1$ we denote the Sobolev space on $S_{h}$, defined by local Lipschitz charts as in Necas [38].

It is to be pointed out that the Sobolev space $H^{r}\left(S_{h}\right)$ (defined in [38]) coincides, with equivalent norms, with the trace space defined in Buffa and Ciarlet in [37] (see also [39] for the case of polygonal boundaries).

When $r>1$, the trace spaces on nonsmooth boundaries can be defined in different ways; we now recall two trace theorems, specialized to our case, referring to [40] and [41] for a more general discussion.

For $f$ in $H^{1}(\mathscr{G})$, we put

$$
\gamma_{0} f(P)=\lim _{r \rightarrow 0} \frac{1}{|B(P, r) \cap \mathscr{G}|} \int_{B(P, r) \cap \mathscr{G}} f(Q) d Q
$$

at every point $P \in \overline{\mathscr{G}}$, where the limit exists. It is known that the limit (A.2) exists at quasi every $P \in \overline{\mathscr{G}}$ with respect to the $(1,2)$-capacity [42].

We now recall the results of Theorem 3.1 in [36] specialized to our case, referring to [41] for a more general discussion.

Proposition A.1. Let $\mathscr{G}$ denote, respectively, $Q, Q_{h}^{1}$, and $Q_{h}^{2}$ and let $\Gamma$ denote $S_{h}, \partial Q_{h}^{1}, \partial Q_{h}^{2}$, and $\partial Q$. Then $H^{1 / 2}(\Gamma)$ is the trace space to $\Gamma$ of $H^{1}(\mathscr{G})$ in the following sense:

(i) $\gamma_{0}$ is a continuous and linear operator from $H^{1}(\mathscr{G})$ to $H^{1 / 2}(\Gamma)$;

(ii) there is a continuous linear operator Ext from $H^{1 / 2}(\Gamma)$ to $H^{1}(\mathscr{G})$, such that $\gamma_{0} \circ$ Ext is the identity operator in $H^{1 / 2}(\Gamma)$.

\section{B. Besov Spaces}

Definition B.1. Let $\mathscr{T} \subset \mathbb{R}^{D}$ be a closed nonempty subset. It is a $d$-set $(0<d \leq D)$ if there exists a Borel measure $\mu$ with supp $\mu=\mathscr{T}$ such that, for some constants $c_{1}=c_{1}(\mathscr{T})>0$ and $c_{2}=c_{2}(\mathscr{T})>0$,

$$
c_{1} r^{d} \leq \mu(B(P, r)) \leq c_{2} r^{d} \quad(P \in \mathscr{T}, 0<r \leq 1) .
$$

Such a $\mu$ is called a $d$-measure on $\mathscr{T}$.

Proposition B.2. The set $F$ is a $d$-set with $d=d_{f}$. The measure $\mu_{F}$ is a $d$-measure. The layer $S$ is a $d$-set with $d=d_{f}+1$. The measure $m$ is a $d$-measure.

See $[23,26]$.

We now come to the definition of the Besov spaces. Actually there are many equivalent definitions of these spaces; 
see, for instance, $[43,44]$. We recall here the one which best fits our aims and we will restrict ourselves to the case $\alpha$ positive and noninteger, $p=q=2$; the general setting is being much more involved; see [44].

Let $\mathscr{T}$ be a $d$-set in $\mathbb{R}^{D}$.

Let $\alpha>0$ be noninteger, $k=[\alpha]$ the integer part of $\alpha$, and $j$ a $D$-dimensional multi-index of length $|j| \leq k$.

If $f$ and $\left\{f^{(j)}\right\}$ are functions defined $\mu$-a.e. on $\mathscr{T}$, we set

$$
R_{j}\left(P, P^{\prime}\right)=f^{(j)}(P)-\sum_{|j+l| \leq k} \frac{f^{(j+l)}\left(P^{\prime}\right)}{l !}\left(P-P^{\prime}\right)^{l},
$$

where $f^{(0)}=f$ and $l$ denotes a $D$-dimensional multi-index. We now define the Besov space as $B_{\alpha}^{2,2}(\mathscr{T}) \equiv B_{\alpha}^{2,2}(\mathscr{T}, \mu)$.

Definition B.3. One says that $f \in B_{\alpha}^{2,2}(\mathscr{T})$ if there exists a family $\left\{f^{(j)}\right\}$ with $|j| \leq k$, as above, such that $f^{(j)} \in L^{2}(\mathscr{T}, \mu)$ and $\left\|\left\{a_{n}\right\}\right\|_{l_{2}}<\infty$, where $a_{n}$ is the smallest number such that

$$
\begin{aligned}
& \left(3^{n d} \iint_{\left|P-P^{\prime}\right|<3^{-n}}\left|R_{j}\left(P, P^{\prime}\right)\right|^{2} d \mu(P) d \mu\left(P^{\prime}\right)\right)^{1 / 2} \\
& \quad \leq 3^{-n(\alpha-|j|)} a_{n} .
\end{aligned}
$$

The norm of $f$ in $B_{\alpha}^{2,2}(\mathscr{T})$ is

$$
\|f\|_{B_{\alpha}^{2,2}(\mathscr{T})}=\|f\|_{2, \mu}+\left\|\left\{a_{n}\right\}\right\|_{l_{2}} .
$$

The family $\left\{f^{(j)}\right\}$ in the previous definition is uniquely determined by $f$, as shown in [44], for $d$-sets with $d>D-1$.

Let us note that for $0<\alpha<1$ the norm $\|f\|_{B_{\alpha}^{2,2}(\mathscr{T})}$ can be written as

$$
\begin{array}{r}
\|f\|_{2, \mu}+\left(\sum_{n=0}^{\infty} 3^{n(d+2 \alpha)} \times \iint_{\left|P-P^{\prime}\right|<3^{-n}}\left|f(P)-f\left(P^{\prime}\right)\right|^{2} d \mu\right. \\
\left.\times(P) d \mu\left(P^{\prime}\right)\right)^{1 / 2} .
\end{array}
$$

Proposition B.4. Let $\mathscr{T}$ be a d-set, $\mathscr{T} \subset \bar{Q}$. Let $s>(3-$ d) $/ 2,(s-(3-d) / 2) \notin \mathbb{N})$; then $B_{s-(3-d) / 2}^{2,2}(\mathscr{T})$ is the trace space to $\mathscr{T}$ of $H^{s}(Q)$ in the following sense:

(i) $\gamma_{0}$ is a continuous linear operator from $H^{s}(Q)$ to $B_{s-(3-d) / 2}^{2,2}(\mathscr{T})$;

(ii) there is a continuous linear operator Ext from $B_{s-(3-d) / 2}^{2,2}(\mathscr{T})$ to $H^{s}(Q)$ such that $\gamma_{0} \circ$ Ext is the identity operator in $B_{s-(3-d) / 2}^{2,2}(\mathscr{T})$.

For the proof, we refer to Theorem 1 of Chapter VII in [44]; see also [43].

From Proposition B.4, it follows that when $\mathscr{T}=S$ and $s=1$ the trace space of $H^{1}(Q)$ is $B_{d_{f} / 2}^{2,2}(S)$.
Let $\beta=d_{f} / 2$. The space $B_{\beta, 0}^{2,2}(S)$ is a subspace of $B_{\beta}^{2,2}(S)$; more precisely

$$
\begin{array}{r}
B_{\beta, 0}^{2,2}(S)=\left\{u \in L^{2}(S, m) \mid \text { there exists } v \in H_{0}^{1}(Q)\right. \\
\text { such that } \left.\gamma_{0} v=u \text { on } S\right\}
\end{array}
$$

equipped with the norm

$$
\begin{gathered}
\|u\|_{B_{\beta, 0}^{2,2}(S)}=\inf \left\{\|v\|_{H^{1}(Q)}: v \in H_{0}^{1}(Q),\right. \\
\left.\gamma_{0} v=u, \text { on } S\right\} .
\end{gathered}
$$

\section{Conflict of Interests}

The authors declare that there is no conflict of interests regarding the publication of this paper.

\section{Acknowledgment}

This research was partially supported under the Grant no. 1109356 by Fractal Fibers and Singular Homogeneization National Science Foundation.

\section{References}

[1] J. Bebernes and D. Eberly, Mathematical Problems from Combustion Theory, vol. 83 of Applied Mathematical Sciences, Springer, New York, NY, USA, 1989.

[2] Y. Giga, "Solutions for semilinear parabolic equations in $L^{p}$ and regularity of weak solutions of the Navier-Stokes system," Journal of Differential Equations, vol. 62, no. 2, pp. 186-212, 1986.

[3] M. R. Lancia, "A transmission problem with a fractal interface," Zeitschrift für Analysis und ihre Anwendungen, vol. 21, no. 1, pp. 113-133, 2002.

[4] M. R. Lancia, "Second order transmission problems across a fractal surface," Accademia Nazionale delle Scienze detta dei XL. Rendiconti. Serie V. Memorie di Matematica e Applicazioni, vol. 27, no. 5, pp. 191-213, 2003.

[5] M. R. Lancia and M. A. Vivaldi, "On the regularity of the solutions for transmission problems," Advances in Mathematical Sciences and Applications, vol. 12, no. 1, pp. 455-466, 2002.

[6] M. R. Lancia and M. A. Vivaldi, "Asymptotic convergence of transmission energy forms," Advances in Mathematical Sciences and Applications, vol. 13, no. 1, pp. 315-341, 2003.

[7] M. R. Lancia and P. Vernole, "Irregular heat flow problems," SIAM Journal on Mathematical Analysis, vol. 42, no. 4, pp. 15391567, 2010.

[8] A. D. Ventcel', "On boundary conditions for multidimensional diffusion processes," Teoriya Veroyatnostei i ee Primeneniya, vol. 4, pp. 172-185, 1959, English translation, Theory of Probability and Its Applications, vol. 4, pp. 164-177, 1959.

[9] H. Fujita, "On the blowing up of solutions of the Cauchy problem for $u_{t}=\Delta u+u^{1+\alpha}$, Journal of the Faculty of Science. University of Tokyo, vol. 13, p. 109-124 (1966), 1966.

[10] H. A. Levine, "The role of critical exponents in blowup theorems," SIAM Review, vol. 32, no. 2, pp. 262-288, 1990.

[11] F. B. Weissler, "Local existence and nonexistence for semilinear parabolic equations in $L^{p}$," Indiana University Mathematics Journal, vol. 29, no. 1, pp. 79-102, 1980. 
[12] F. B. Weissler, "Existence and nonexistence of global solutions for a semilinear heat equation," Israel Journal of Mathematics, vol. 38, no. 1-2, pp. 29-40, 1981.

[13] F. B. Weissler, "Semilinear evolution equations in Banach spaces," Journal of Functional Analysis, vol. 32, no. 3, pp. 277296, 1979.

[14] F. Gazzola and T. Weth, "Finite time blow-up and global solutions for semilinear parabolic equations with initial data at high energy level," Differential and Integral Equations, vol. 18, no. 9, pp. 961-990, 2005.

[15] K. Falconer and J. Hu, "Nonlinear diffusion equations on unbounded fractal domains," Journal of Mathematical Analysis and Applications, vol. 256, no. 2, pp. 606-624, 2001.

[16] M. Matzeu, "Mountain pass and linking type solutions for semilinear Dirichlet forms," in Recent Trends in Nonlinear Analysis, vol. 40 of Progress in Nonlinear Differential Equations and Their Applications, pp. 217-231, Birkhäuser, Basel, Switzerland, 2000.

[17] A. Grigor'yan, J. Hu, and K.-S. Lau, "Heat kernels on metric measure spaces and an application to semilinear elliptic equations," Transactions of the American Mathematical Society, vol. 355, no. 5, pp. 2065-2095, 2003.

[18] M. R. Lancia and P. Vernole, "Semilinear evolution transmission problems across fractal layers," Nonlinear Analysis, vol. 75, no. 11, pp. 4222-4240, 2012.

[19] M. R. Lancia and P. Vernole, "Semilinear fractal problems: approximation and regularity results," Nonlinear Analysis, vol. 80, pp. 216-232, 2013.

[20] M. Cefalo, G. Dell'Acqua, and M. R. Lancia, "Numerical approximation of transmission problems across Koch-type highly conductive layers," Applied Mathematics and Computation, vol. 218, no. 9, pp. 5453-5473, 2012.

[21] M. R. Lancia and P. Vernole, "Convergence results for parabolic transmission problems across highly conductive layers with small capacity," Advances in Mathematical Sciences and Applications, vol. 16, no. 2, pp. 411-445, 2006.

[22] K. Falconer, The Geometry of Fractal Sets, Cambridge University Press, Cambridge, UK, 2nd edition, 1990.

[23] U. Freiberg and M. R. Lancia, "Energy form on a closed fractal curve," Zeitschrift für Analysis und ihre Anwendungen, vol. 23, no. 1, pp. 115-137, 2004.

[24] U. Mosco, "Lagrangian metrics on fractals," in Proceedings of Symposia in Applied Mathematics, R. Spigler and S. Venakides, Eds., vol. 54, pp. 301-323, American Mathematical Society, 1998.

[25] M. Fukushima, Y. Oshima, and M. Takeda, Dirichlet Forms and Symmetric Markov Processes, vol. 19 of de Gruyter Studies in Mathematics, Walter de Gruyter, Berlin, Germany, 1994.

[26] U. Mosco and M. A. Vivaldi, "Variational problems with fractal layers," Accademia Nazionale delle Scienze detta dei XL. Rendiconti. Serie V. Memorie di Matematica e Applicazioni, vol. 27, no. 5, pp. 237-251, 2003.

[27] T. Kato, Perturbation Theory for Linear Operators, Springer, Berlin, Germany, 2nd edition, 1977.

[28] R. Rammal and G. Tolouse, "Walks on fractal structures and percolation clusters," Journal de Physique Lettres, vol. 44, pp. L13-L-22, 1983.

[29] M. Fukushima and T. Shima, "On a spectral analysis for the Sierpiński gasket," Potential Analysis, vol. 1, no. 1, pp. 1-35, 1992.

[30] E. B. Davies, Heat Kernels and Spectral Theory, Cambridge University Press, Cambridge, UK, 1989.

[31] T. Kumagai, "Brownian motion penetrating fractals," Journal of Functional Analysis, vol. 170, no. 1, pp. 69-92, 2000.
[32] A. Grigor'yan, "Heat kernel upper bounds on a complete noncompact manifold," Revista Matemática Iberoamericana, vol. 10, no. 2, pp. 395-452, 1994.

[33] U. Mosco, "Convergence of convex sets and of solutions of variational inequalities," Advances in Mathematics, vol. 3, pp. 510-585, 1969.

[34] U. Mosco, "Composite media and asymptotic Dirichlet forms," Journal of Functional Analysis, vol. 123, no. 2, pp. 368-421, 1994.

[35] C. Baiocchi and A. Capelo, Variational and Quasivariational Inequalities: Applications to Free-Boundary Value Problems, John Wiley \& Sons, New York, NY, USA, 1984.

[36] D. Jerison and C. E. Kenig, "The inhomogeneous Dirichlet problem in Lipschitz domains," Journal of Functional Analysis, vol. 130, no. 1, pp. 161-219, 1995.

[37] A. Buffa and P. Ciarlet," "On traces for functional spaces related to Maxwell's Equations-part I: an integration by parts formula in Lipschitz Polyhedra," Mathematical Methods in the Applied Sciences, vol. 21, no. 1, pp. 9-30, 2001.

[38] J. Necas, Les mèthodes directes en thèorie des èquationes elliptiques, Masson, Paris, France, 1967.

[39] F. Brezzi and G. Gilardi, "Foundamentals of P.D.E. for numerical analysis," in Finite Element Handbook, H. Kardestuncer and D. H. Norrie, Eds., McGraw-Hill, New York, NY, USA, 1987.

[40] P. Grisvard, Elliptic Problems in Nonsmooth Domains, vol. 24, Pitman, Boston, Mass, USA, 1985.

[41] P. Grisvard, "Théorèmes de traces relatifs à un polyèdre," Comptes Rendus de l'Académie des Sciences A, vol. 278, pp. 15811583, 1974.

[42] D. R. Adams and L. I. Hedberg, Function Spaces and Potential Theory, Springer, Berlin, Germany, 1966.

[43] H. Triebel, Fractals and Spectra related to Fourier Analysis and Function Spaces, vol. 91 of Monographs in Mathematics, Birkhäuser, Basel, Switzerland, 1997.

[44] A. Jonsson and H. Wallin, Function Spaces on Subset of $\mathbb{R}^{n}$ , vol. 2, part 1 of Mathematical Reports, Harwood Academic Publishers, London, UK, 1984. 


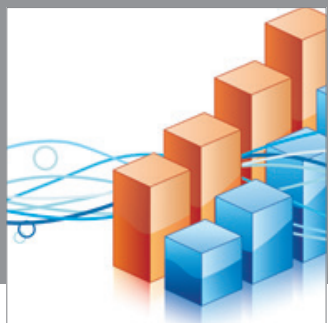

Advances in

Operations Research

mansans

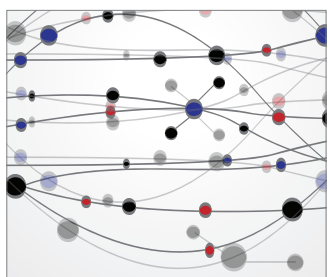

The Scientific World Journal
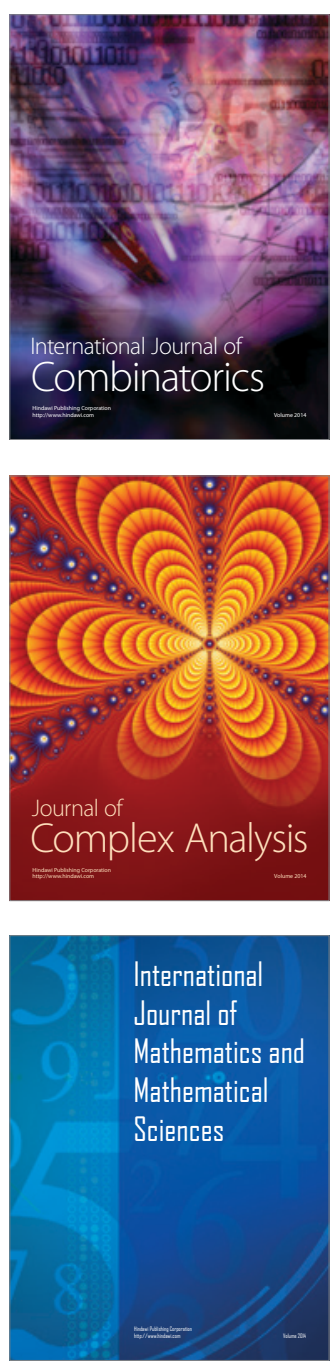
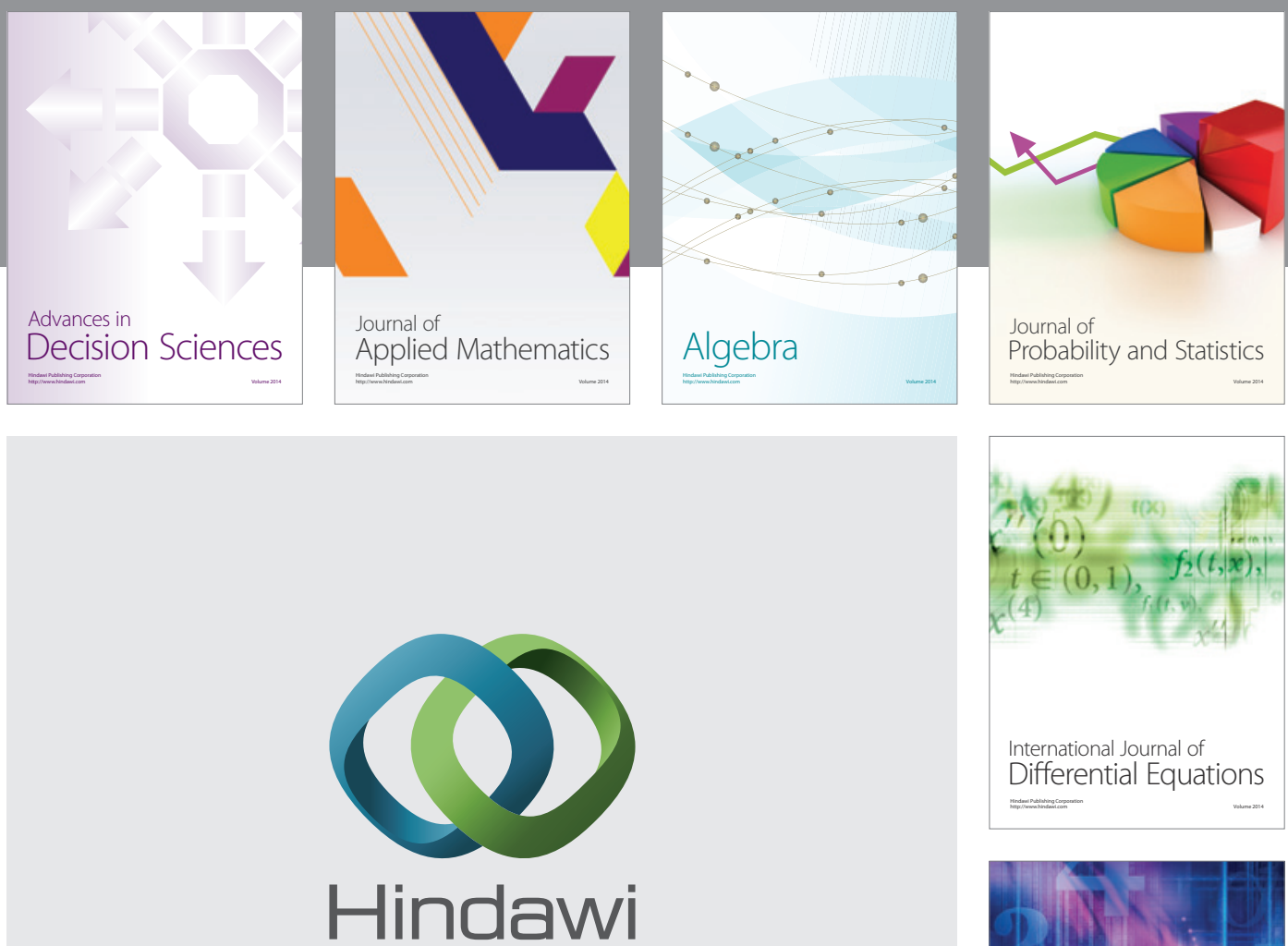

Submit your manuscripts at http://www.hindawi.com
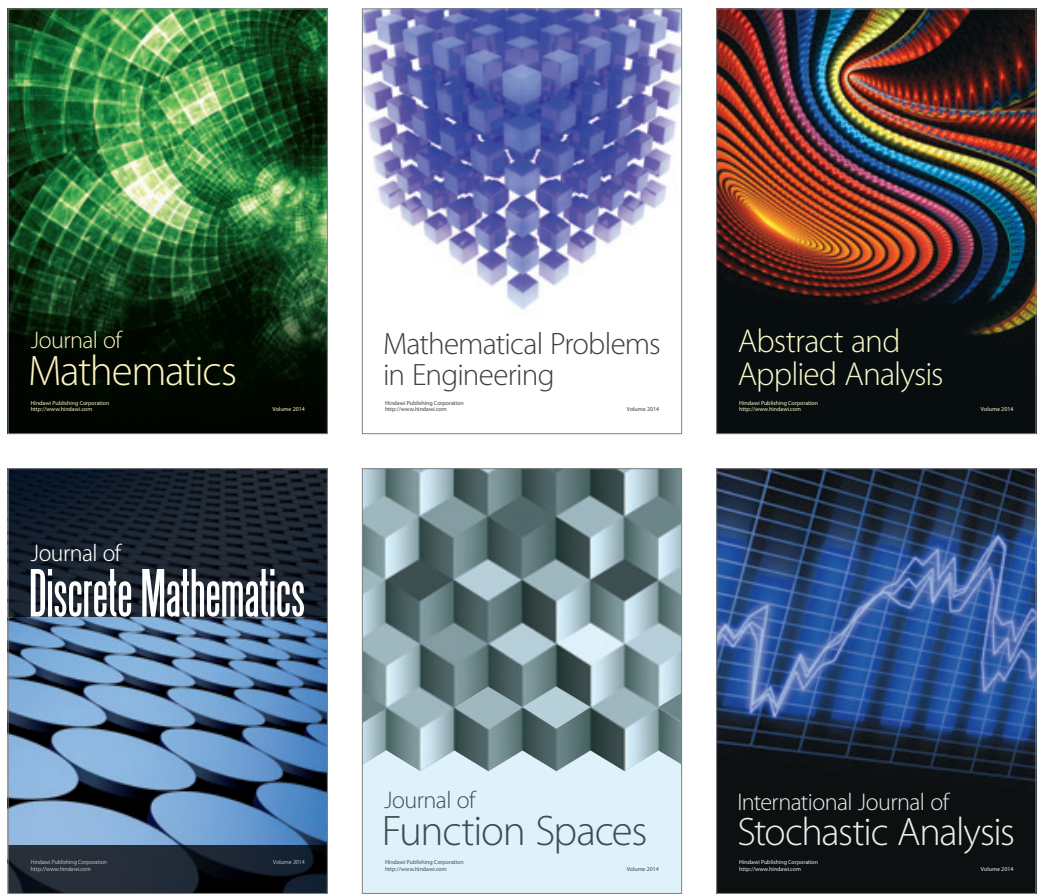

Journal of

Function Spaces

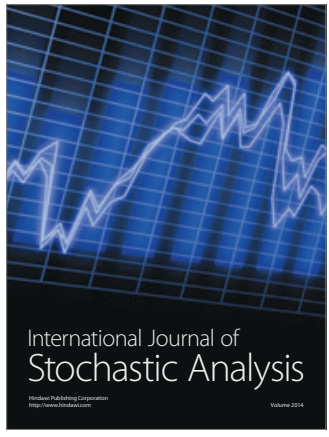

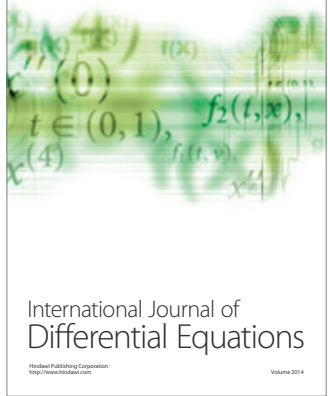
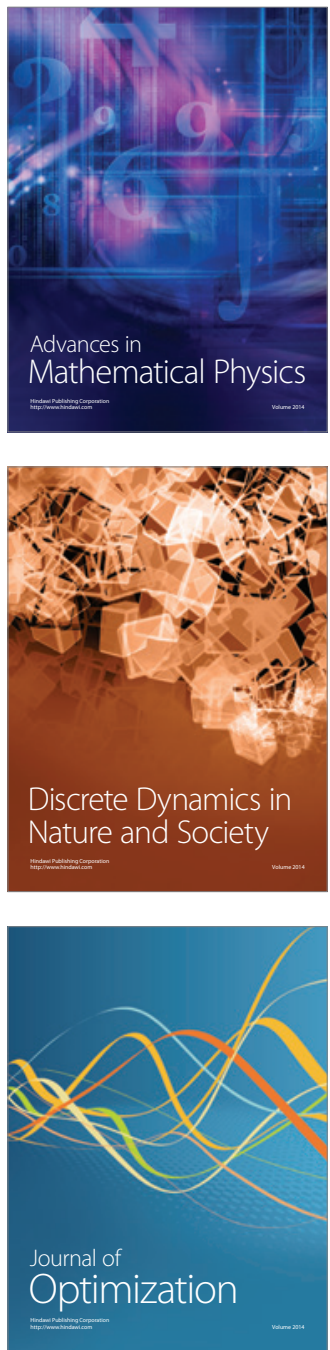